\title{
Le nom propre dans la tragédie shakespearienne
}

\section{Ann Lecercle}

\section{(2) OpenEdition \\ Journals}

\section{Édition électronique}

URL : http://journals.openedition.org/shakespeare/1328

DOI : 10.4000/shakespeare.1328

ISSN : 2271-6424

Éditeur

Société Française Shakespeare

Édition imprimée

Date de publication : 1 novembre 1989

Pagination : 241-258

\section{Référence électronique}

Ann Lecercle, "Le nom propre dans la tragédie shakespearienne ", Actes des congrès de la Société française Shakespeare [En ligne], 7| 1989, mis en ligne le 01 janvier 2007, consulté le 20 avril 2019. URL : http://journals.openedition.org/shakespeare/1328; DOI : 10.4000/shakespeare.1328 


\title{
Société Française Shakespeare
}

\author{
Actes des Congrès \\ 1985 - 1986 - 1987
}

\section{LA FOLIE}

\section{SHAKESPEARE ET LES ARTS \\ LE TRAGIQUE}




\title{
LE NOM PROPRE DANS LA TRAGEDIE SHAKES- PEARIENNE
}

\author{
Ann LECERCLE
}

\begin{abstract}
Dans un ouvrage intitulé Noms Propres ${ }^{1}$, Emmanuel Lévinas pose la question liminaire : à une époque marquée, selon lui, par la «hantise de l' inexprimable, de l' ineffable, du non-dit dans le mal-dit», en un mot, par «la rupture douloureuse du discours»2n, les noms propres constituent-ils le «dernier bastion du Dit face au Dire» ${ }^{2 b}$, le dernier refuge de l'Absolu, de la transcendance, de la séparation, pour tout dire de l'intouchable de la langue ? S'agit-il là d'une vision judaïque du langage, fondée sur le Nom Imprononçable, donc effectivement hors d'atteinte de toute entreprise de sape? Sans doute.

Car il y a toujours eu une catégorie de textes fondateurs où le nom propre peut représenter tout le contraire d'un roc inébranlable, devenant au contraire la clé de voûte d'une architecture verbale qui se trouve être, non moins que la Bible, l'une des références de base de la culture occidentale. Cette catégorie, c'est la tragédie. Or, comme l'a fait remarquer George Steiner dans Antigones, en dépit de «its millenial immersion in suffering, [the judaic sensibility] does not produce tragic drama [because] Judaism incarnates this abandonment of man's inmost "to an alien

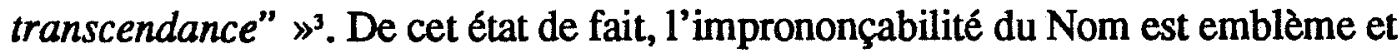
symptôme. Or c'est par le nom juif, Aaron dans Titus Andronicus, que Shakespeare commence le nom pour en faire une image du destin du personnage.

Rappelons brièvement le cas d'Edipe $R o i$, qui représente aux yeux d'Aristote le paradigme du genre. Du héros éponyme le nom, on le sait, a deux faces : oida (je sais)bidos pous (pied enflé) 4 . Car ce signifiant donne à entendre la division du sujet, égal aux dieux (isotheos), Jégal aux bêtes, (pharmakos). Cette division, cette Spaltung, programme la péripétie qui produit le passage de la méconnaissance à la reconnaissance (anagnorisis), sur lesquelles repose la structure «énigmatique» de la tragédie. Signalons aussi, car il en sera maintes fois question par la suite, le jeu entre la quasi-totalité du savoir supposé (oida) et le petit bout de chair (dont Lacan dira qu'il est «imbécile») qui manque à l'ensemble et qui finit néanmoins par abolir tout le restes (oidos pous). Bref, on peut dire que d'une certaine façon, le texte entier est
\end{abstract}


contenu en germe dans le nom. Si tel est le cas, c'est qu'à la différence du sujet biblique, le sujet de la tragédie n'est pas source du signifiant, il est refendu par lui, et cela jusque dans son nom.

Ainsi, si le nom propre est le roc incontournable de la langue, ce roc, dans la tradition grecque, est destiné à être clivé en deux, sinon par les foudres de Dieu, du moins par le Logos oraculaire et énigmatique des dieux - ce qui, dans la tragédie, revient au même.

Dans Shakespeare comme dans Sophocle, le nom occupe une place à part dans le dispositif tragique. Un rapide survol suffit à s'en convaincre : dans Richard III, l'hécatombe de victimes innocentes commence quand Clarence va à sa mort parce que son nom commence par la lettre G ; dans Jules César, on fait mieux, on tue le nom à la place de la personne, à titre d'exemple Cinna le poète au lieu de Cinna le conspirateur. Au centre d'Othello est la représentation que Shakespeare baptise «unlacing», délacement, du nom. Tant et si bien qu'au lever du rideau dans Macbeth on est d'emblée plongé dans un univers étrange où les actes n'ont carrément plus de nom, un univers de «deeds without names». Il n'est pas surprenant dans ces circonstances que les deux principaux acteurs, bien avant la fin de la pièce, ne sont plus désignés que par des périphrases en lieu et place du nom propre. Coriolan va plus loin encore : non seulement on ne trouve plus de nom qui lui convienne - c'est «a thing», voire «a no-thing» - il interdit qu' on en use, et jusqu'au titre de l'œuvre devient un affront mortel, une invitation à tuer celui qui le porte en nom, une provocation à la boucherie :

\section{"Coriolanus"}

He would not answer to ; forbad all names: He was a kind of nothing, titleless

$$
(\mathrm{V}, \mathrm{i}, 11-13) \text {. }
$$

[...] Ay, Martius, Caius Martius ! Dost thou think

I'll grace thee with that robbery, thy stol' $n$ name. Coriolanus in Corioles?

$$
(\mathrm{V}, \mathrm{vi}, 88-89)^{6}
$$

Ce n'est pas un détail superflu, ni même simplement symbolique : le nom est la clé de voûte du fonctionnement du langage dans la tragédie. Ceci pour autant que le langage repose sur le nomos, c'est-à-dire, avant même qu'il ne signifie la loi, le geste du paysan qui délimite un territoire. Or, dans la tragédie, la délimitation du 
territoire est entamée par deux figures complémentaires : $d$ 'unepart,l'équivocation, qui fait de la tragédie le genre par excellence de l'énigme ; de l'autre, celle qu'Aristote plaçaitau centre de la réthorique tragique, l'amphibolie (on pourrait dire que dans Macbeth, le jeu de la paronomase jointe à l'oxymore - guilt/gilt, breach/ breech, shoal/school - représente sans doute ce qu'il y a de plus proche dans Shakespeare de l'amphibolie sophocléenne). Après tout, si à l'origine du thêatre occidental on trouve la tragédie, c'est que, comme on a pu l'écrire récemment,

[...] ce que nous aimons au thêâtre ? C"est peut-être que chaque mise en scène démontre comme la même chaîne signifiante est pliable, et qu' il n'est monument ou langage qu' équivocation et amphibolie ne corrodent, ne métamorphosent $»^{?}$.

En d'autres termes, le télescopage du proche et du lointain ne concerne pas que le territoire politique, ni même institutionnel, il concerne le territoire sémantique dans son ensemble. L'adage des Weird Sisters, «Fair isfoul, and foul is fair», prend à cet égard une valeur exemplaire. Le geste du paysan instituant la délimitation territoriale joue jusque dans la substance de la langue et de la vie, en l'occurrence dans le sexe, dans le partage sexuel entre femmes et entre générations. Comme l'a pu écrire Catherine Clément, là où il y a énigme, il y a inceste - et vice versa - ou, à tout le moins, sinon un manque de distance, un excès, que ce soit le bannissement de Cordelia ou le noctambulisme de Lady Macbeth, Nomos. Le geste du paysan, la trace de la coupure sur la terre, $c$ 'est aussi le geste du père qui interdit la mère. C'est pourquoi le nom propre est le Nom-du-Pere .

Or, dans la tragédie de Shakespeare, le nom propre se dessine comme le lieu d'une éclipse. Elle est provoquée par le jeu du signifiant onomastique et elle aboutit à oblitérer le Nom-du-Père, pour promouvoir à sa place le plus monstrueux des noms : ce qu'il faut bien appeler, tel qu'il est livré par la configuration du texte, le Nom-de-la-Mère, ou, à défaut, le nom inscrit dans la chair. Le passage de l'un à l'autre est une manière de chute. Cette chute est esquissée en filigrane dans une scène qui joue un rôle charnière dans l'œuvre de Shakespeare. En effet, àl'orée des grandes tragédies, dès la toute première scène d'Hamlet, Shakespeare semble ébaucher une version corporelle, liminaire, paradigmatique, de cette chute. Il s'agit du long récit d'Horatio évoquant la fin de l'Age d'Or de la Chevalerie et du respect de la parole donnée. Lardé de termes techniques du droit, ce discours raconte le combat loyal de deux preux chevaliers. Quand il en vient au présent età l'entreprise déloyale du jeune Fortinbras, on passe du nomosi - du domaine de la Loi et de la distribution des terres - à un tout autre univers conceptuel, et plus encore fantasmatique. S'inscrit alors en filigrane dans le discours politique et moral d'Horatio une vision de jupes, de ventre, de boire et de manger, d'une gueule béante et dentée :

\section{[...] Yound Fortinbras}


Hath in the skirts of Norway here and there

Shark'd up a list of lawless resolutes

For food and diet to some enterprise

That hath a stomach in' $t$

$(\mathrm{I}, \mathrm{i}, 99-103)^{8}$.

Le chaudron des sorcières dans Macbeth ne représente pas autre chose.

Dans Lear aussi, au lever du rideau, on retrouve la même logique, mais inversée : à savoir, les jupes emblématiques d'une configuration, d'une imago, maternelle, cette fois bonne et nourricière, protectrice non prédatrice, qui se profile cette fois à travers le brutal démembrement du territoire au lieu du remembrement du territoire dans le respect du droit, qui sert de toile de fond au discours d'Horatio :

Of all these bounds, even from this line to this,

With sahadowy forests and with champains rich'd,

With plenteous rivers and wide-skirted meads,

We make thee lady

$$
(\mathrm{I}, \mathrm{i} ., 63-6)^{9} \text {. }
$$

Le geste de Lear ici est le geste même du paysan grec, du nomos qui trace les limites du territoire, de ce qui «échoue en partage», pour citer le Bailly. Seulement c'est un travestissement du Nom-du-Père, une négation du nomos, car ce qui est partagé, démembré et même «digéré» ("With my two daughters' dowers digest the third», I, i., 128) entre les gendres, c'est une terre investie de tous les traits, pour ne pas dire attraits, - fertilité et protection - du corps de la mère, c'est-à-dire celle qui, comme l'ont toujours démontré les ethnologues, doit demeurer, au sein de la tribu, la plus éloignée des gendres. On pourrait dire que le tableau liminaire duRoi Lear avec ce sparagmos et le repas totémique, perverti parce que maternel qui s'ensuit est un renversement du tableau final des Bacchantes d'Euripide, où c'est la mère, Agavé, qui pratique le sparagmos sur le corps de son fils, le donnant à ingérer aux femmes qui l'accompagnent dans sa folle course.

Pour revenir à Hamlet et Lear, on peut donc constater la présence d'un diptyque liminaire d'une grande prégnance : $d$ 'une part, le remembrement du territoire selon le droit, nomos au nom de la Loi et d'un Père idéal, qui refoule une imago maternelle effrayante, contenant en germe le chaudron des sorcières, de l'autre, le démembrement du territoire sous le signe d'une image maternelle parée de tous les attraits de la chair. Bref, ce diptyque symétrique mis en place chaque fois dès le lever du rideau donne à voir, comme en exergue, le rapport entre nomos, Nomdu-Père et imago de la Mère ; entre nom et image, entre terre et chair, quand l'effacement du Nom-du-Père, se profile ce que j'ai baptisé le nom monstrueux de la Mère. 
J'ajouterai enfin, pour clore cette introduction, que si, dans Shakespeare, la tragédie atteint l'un de ses sommets, c'est en partie que le jeu de miroirs entre macrocosme et microcosme, entre corps politique et corps naturel, produit une imbrication entre terre et chair, entre idéologie et institutions d'une part, et entre désir et fantasme de l'autre, d'une complexité et d'une richesse mais aussi d'une cohérence rarement égalées. Si bien que c'est véritablement mutiler ce qu'il y a de plus précieux dans l'œuvre que de prétendre faire l'impasse sur l'un des deux registres, la chair sans la terre autant que la terre sans la chair, le fantasme sans l'idéologie, l'idéologie sans le fantasme.

C'est par Lear que je propose de commencer. Mais pas avant de faire un bref détour par un lointain ancêtre, Titus Andronicus. Car le destin d'Aaron, Maure au nom étrangement juif, offre un modèle réduit de ce que l'on retrouvera plus tard dans les tragédies de la maturité, où le jeu onomastique concernera les personnages principaux. Dans le cas d'Aaron, amené à Rome dans les bagages des Goths captifs en tant qu'amant attitré de leur reine Tamora, la dérive du nom est aussi le destin du personnage. Cet être, où s'affiche ce qui, aux yeux des Elisabéthains, paraissait une triple monstruosité - barbare, noir et juif - est celui par qui le malheur arrive : les derniers mots de l'œuvre sont les suivants :

See justice done on Aaron, that damn'd Moor, By whom our heavy haps had their beginning ; Then, afterwards, to order well the state, That like events may ne' er it ruinate. (Exeunt). Finis..$^{10}$

Il y a chez ce personnage une espèce de splendeur sinon de grandeur dans la perversion, une joie et une santé insolente dans la malfaisance : c'est à tel point le cas qu'au seuil de la mort, Aaron assume le mal qu'il incarne en prenant bien soin de renier les rares bonnes actions qu'il risque - purement par mégarde - d'avoir commises pendant sa vie. Shakespeare l'investit d'une lucidité et d'une vigueur qui le font échapper à son rôle traditionnel de «Vice». Même s'il n'est pas le héros en titre de l'œuvre, face à la décadence de l'Empire tardive, ainsi qu'à un héros affublé systématiquement de l'épithète «vieux», «the old Andronicus» (ce qui finit par friser le comique), Aaron en vient à occuper cette place vide plus habituellement réservée au personnage héroïque. C'est un Iago avant la lettre, mais un Iago noir, doué du pouvoir de séduction d'un Othello.

Or, le châtiment - la «justice faite», pour citer les vers ci-dessus - de ce criminel hors du commun est doublement à l'image de la transgression : comme pour Iago, il prend une tournure particulière ; la mort rapide étant d'une trop grande douceur pour l'occasion, il doit, comme le dira en toutes lettres Hamlet dans la première des grandes tragédies, «manger l'air» («I eat the air, promise-crammed. 
You cannot feed capons so. III, ii, 93-4) ${ }^{18}$. En clair, Aaron est condamné à mourir de faim ${ }^{19}$. Châtiment particulièrement apte, car s'il n'a pas lui-même transgressé oralement (par cannibalisme assaisonné d'infanticide), d'autres l'ont fait pour venger les méfaits par lui commis. Or, ces méfaits atteignent leur point culminant à l'acte IV, où on le remercie de ses boucheries - les dernières qu'il aura l'occasion de commettre, car il ne réapparaîtra que captif - dans deux vers dont il importe de ne pas sous-estimer la symétrie chiastique :

$\begin{array}{ll}\text { Chiron : } & \text { Aaron, I see thou wilt not trust the air } \\ \text { With secrets. } \\ \text { For this care of Tamora, } \\ \text { Demetrius : } \quad \begin{array}{l}\text { Herself and hers are highly bound to thee. } \\ \text { (Exeunt Dem. And Chi. bearing off the Nurse's body) }\end{array}\end{array}$

(IV, ii, 169).

Ce discours est énoncé au-dessus du cadavre de la nourrice du fils nouveauné suppliciée quelques instants auparavant par Aaron,-afin que, dans les termes du texte, l'air ne la trahisse pas. Or l'ironie dramatique (et la Justice divine) font que l'objet auquel est ravalé le nom juif, $1^{\prime}$ 'Aaron ${ }^{11}$ du début du vers devenant l'air de la fin du vers, est justement destiné à être l'objet oral par excellence lors du supplice, quand ce sera sa seule et unique nourriture. D'ailleurs, la scène du supplice est marquée par l'inattendu surgissement ambigu breast (sein/poitrine) : «Set his breast-deep in earth and famish him». On a là comme une inversion mortifere du tableau de la-mère-nourricière-et-l'enfant, comme le sera de manière beaucoup plus insidieuse et omniprésente, toute la problématique de l'invidia dans All's Well et Othello (1603-4). Rien à voir par exemple avec le modèle médiéval du supplice de la faim, c'est-à-dire la mort du Comte Ugolino enfermé avec ses enfants dans une tour, dans l'avant-dernier cercle de l'Enfer de Dante.

Toujours aussi lucide, Aaron lui-même ne manque pas de souligner ce que cette mise à mort comporte de retour pervers au sein nourricier. C'est cette représentation paternelle qui lui servira d'exorde au discours qui précède l'enterrement partiel, où c'est d'enfance au sens propre comme au sens figuré - infantis, mutisme - qu'il s'agit :

$O$, why should wrath be mute, and fury dumb?

I am no baby, I, that with base prayers

I should repent the evils I have done

( $V$, iii, 183-5, nous soulignons).

Avec ces mots se ferme une boucle qui contient un des noyaux de la représentation dans la pièce, car si la subversion du nom s'achève sur l'image d'un bébé, elle commence de la même manière. En effet, si la Nourrice est tuée, si Aaron 
«will not trust the air», c'est que la Nourrice a vu naitre un bébé, fils d'Aaron et de Tamora. L'enterrement jusqu'à la poitrine dans la terre, pour qu'Aaron hurle de faim, est la représentation inverse et complémentaire, la négation de cette naissance, la projection du film à rebours en quelque sorte l'engloutissement dans la TerreMère.

Ainsi se produit une péripétie onomastique, un retournement provoqué par le ravalement du nom (Aaron) en objet oral mortifêre (air), où, comme un enfant au sein, celui qui ne voulait pas se fier à l'air doit s'en remettre à l'air pour vivre. Il y a dans cette mise à mort une castration du nom symboliquement privé de sa queue comme, lors du supplice, s'effacera la moitié inférieure du corps («breast-deep»). Dans cette éclipse du Nom-du-Père ce qui se venge, c'est autant la chair de la TerreMère que l'air dans Aaron.

A cet égard, Coriolan, la dernière des tragédies, renoue avec Titus, la première. On a pu écire, à juste titre, que «Coriolan est [...] l' histoire tragique d' un nom» (Philip Brockbank) ${ }^{12}$. Seulement il est nécessaire de s'entendre sur le sens à donner à cette formule. Il ne s'agit pas simplement d'inscrire la problématique du nom dans Coriolan dans une perspective de psychologie morale où renommée est synonyme d'immortalitétis. Shakespeare l'aborde sous des angles très divers, où idéologie et fantasme sont étroitement imbriqués, comme par exemple dans l'incident entièrement inventé par lui où le héros n'arrive pas à se souvenir du nom de son sauveur dans la ville ennemie.

Pour Coriolan comme pour Aaron, le destin du personnage est aussi le destin du nom. En effet, la mise à mort du héros prend la forme d'un quasi rituel qui commence de manière identique : on coupe au personnage un bout de son nom. Il ne lui reste alors plus que la moitié d'un nom, «Caius Martius». Mais l'aristocrate romain n'est pas le captif barbare: comme William de la Pole lors de sa chute «tragique» dans Henri VI 2, il effectue un renversement in extremis de la castration onomastique : l'aristocrate anglais efface alors Pole comme pool (égout) par Pole comme trophée, c'est-à-dire comme poll sur un pole (tête sur un pieu). Non moins aristocrate que ce dernier, une fois dépouillé de son agnomen, Coriolan, ravià la ville étrangère (Corioles), il re-sémantise le moignon qui lui reste (Caius Martius) en faisant surgir non le nom de la gens mais le dieu Mars lui-même :

«Hear'st thou Mars?» Aufidius«Name not the god, thou boy of tears !» (V, vi, 99101). Si le héros a dû pousser son Familienroman jusqu'à ces extrémités divines, c'est que cette invocation du nom du dieu est la réplique à une remarque d'Aufidius qui met à nu la représentation commune qui est sous-jacente aux supplices du juif noir et du romain renégat - à savoir le spectacle des hurlements de l'homme redevenu enfant, voire nourrisson face à la nourrice, aussi présente ici que dans 
Titus. En effet, les paroles qui précèdent immédiatement l'invocation de Mars sont les suivantes:

\section{But at his nurse's tears \\ He whin' $d$ and roar' $d$ away your victory, That pages blush' $d$ at him, and men of heart \\ Look'd wond' ring each at others.}

$$
\text { (V, vi, 97-100) }
$$

Whine associéà roar évoque, plus encore que le «gamin» (boy) d'Aufidius, le spectacle de la naissance de Gargantua, ou de la mort d'Aaron, hurlant de faim. Pourquoi ?

Coriolan n'est pas un comparse comme Aaron. Aussi les soubassements fantasmatiques sont-ils dans cette tragédie beaucoup plus structurés, et occupent, autant que les considérations politiques, le premier plan de la représentation. Pour répondre à notre question il convient de se reporter en arrière, aux principes de la nomination, comme à la scène où le héros éponyme a été «baptisê» où le fantasme se laisse presque voir.

Si on prend comme modèle le cas de Publius Cornelius Scipio Africanus, on distingue dans l'ordre le praenomen, nom de l'individu, le nomen, nom de la gens, de la «famille étroite», et enfin l'agnomen, le nom de l'endroit où s'est accompli l'exploit héroïque. Dans le cas qui nous intéresse, Caius correspond au prénom, Martius au nom de la gens; quant à celui qui, en cours de route, viendra s'y accoler, foumissant le titre de l'auvre, c'estl'agnomen qui supplée au manque de cognomen. Ce manque existe déjà dans la source. Mais la pièce que Shakespeare a écrite à partir de Plutarque l'exploite de façon magistrale Coriolan, c'est la forclusion du Nom-duPere.

En soi, l'absence de cognomen n'est pas totalement anormale; mais s'agissant d'une famille illustre, elle le devient. Chez Shakespeare, ce manque éminemment symptomatique est aussitôt renforcé par trois autres manques symboliques dus entièrement à la plume du dramaturge :

a) les dieux, s'ils ne sont pas absents, se sont du moins retirés à une distance olympienne des événements qui se déroulent devant nos yeux ;

b) les ambassades, chez Plutarque, sont au nombre de quatre : Shakespeare en supprime une, la troisième - c'est le troisième nom qui disparait -celle des grands prêtres de Rome ;

c) étrange latinité, étrange aristocratie : le père lui-même est totalement inexistant dans la pièce de Shakespeare (bien plus, par exemple, que la mère dans la famille Lear) ;

d) autre détail introduit par Shakespeare, 1'oubli du nom de celui à qui notre héros «doit la vie», non pas son père, mais son sauveur, lorsqu'il était en danger dans la ville ennemie : «Martius, hisname? ?/»Forgot, by Jupiter !» $(\mathrm{I}, \mathrm{ix}, 88)^{14}$. 
Il y a là un quadruple affaiblissement des instances du symbolique. A quoi vient répondre le scénario tout à fait particulier de l'exploit héroïque et de l'imposition du nouveau nom. En dernière analyse, la stratégie de la mère du héros, Volumnia, lorsqu'elle envoie son fils à peine sorti des langes, sur le champ de bataille, est de re-sémantiser le nomen, Martius, en faisant de son fils le fils non de son père mais du dieu de la guerre, Mars.

Remplacer le géniteur par le destructeur, faire de son fils le fils non de ses parents mais de l'accouplement entre Mars et l'Urbs, la cité à investir, à «prendre» à "conquérir» : tel est l'objectif de Volumnia. Coriolanus, c'est ainsi le nom de l'Autre - de l'Etrangère - violée. En effet, l'une des images les plus impressionnantes de tout le corpus shakespearien est l'apparition de Martius, dont on croyait que, étant entré seul dans Corioles, il serait «passé à la marmite» : «To th' pot, I warrant him» (I, iv, 47), comme disent les soldats romains. Deux ans auparavant, Shakespeare nous l'avait montrée, cette marmite : c'était, dans Macbeth le chaudron des sorcières, où, dans le feu et le sang, on confectionnait des êtres à partir de morts ou de morts nés. Car dans la marmite qu'est Corioles livrée au glaive, Coriolan renaît de la mort en emportant avec lui le nom de cette marmite, matricielle et mortuaire à la fois, ayant préalablement et explicitement «oublié» le nom de celui qui, làdedans, fut son protecteur, celui à qui il doit la vie ; autrement dit, il scotomise le signifiant paternel dans le ventre de la mère.

Car l'appartition apocalyptique de cet être aussi méconnaissable qu'innommable devant les portes de Corioles, n'est pas simplementl'image traditionnelle du guerrier «couvert de sang» : il est sang de la tête aux pieds ; comme Marayas, il paraît écorché vif, car on le reconnaît, comme Gargantua sortant du ventre de sa mère, uniquement au son de sa voix de stentor incontrôlée car incontrôlable. Cette image est surtout une image de jouissance dans les entrailles sanglantes d'une ville violée. Dans Macbeth, Shakespeare nous donne le point de vue de la ville : on songe en effet à l'étrange délectation de Lady Macbeth à la pensée de Duncan prêt à entrer «under my battlements», comme si la forteresse et son corps ne faisaient qu'un, et où Duncan aussi, à sa façon, va «passer à la marmite». C'est que, tant dans l'enceinte du château écossais que de la ville latine, le temps est en proie à l'horreur, cette «horreur du temps» dont parle Macbeth, alors qu'il se compare à Tarquin se préparant à violer Lucrèce. S'il y a horreur, c'est que passé et avenir se télescopent dans un présent qui n'est autre que le temps par excellence du fantasme (lequel se définit, dit Lacan, comme le futur antérieur). Ainsi, cette sortie de Corioles aussi stridente que sanglante, est elle aussi une atroce naissance. Et l'agnomen formé à partir de ce corps violé et parturient, c'est le Nom, non du Père, mais de la Mère : Coriolanus, de Corioles, qui, prise et fécondée, accouche aussitôt, dans ce magma temporel pétrifié, d'un être proprement innommable, rapidement destiné à devenir explicitement «une chose», «auteur de lui-même», sans nom.

C'est aussi la contamination par le nom monstrueux de la Mère qui provoque un phénomène singulier (dans lequel on a souvent voulu voir une erreur textuelle) : lors de la cérémonie de l'imposition solennelle de l'agnomen à Rome, les 
deux noms hérités à la naissance, Caius Martius, sont tout à coup pris d'un bien étrange vertige, car, accouplés à «Coriolanus», praenomen et nomen par deux fois changent de place :

For what he did before Corioles, call [...]

Martius Caius Coriolanus (sic)

Bear the addition nobly ever!

All : Martius Caius Coriolanus

$$
\text { (I, ix, 61-6). }
$$

C'est qu'on ne peut accoupler le Nom-du-Père et ce qui est en fait, grấce au scénario fantasmatique qui sous-tend la guerre dans la pièce, le nom d'un viol incestueux, où la mère toute proche devient l'Etrangère par excellence, celle qu'on viole mais aussi le corps de l'Autre dont on naît. Une telle cohabitation est impossible à concevoir ou alors le corps en est déchiré : «Tear him to pieces».

L'ironie de Coriolan, c'est que ce fils, dont la mère a trop voulu le re-nom, finit non seulement sans nom mais sans corps : s'il n'est pas déchiré vivant, mort, il est aussitôt ravalé au niveau d'une carcasse ou d'un déchet, et si aucun autre héros tragique de Shakespeare ne connaît un Familienroman aussi hubristique, nul autre non plus ne tombe si bas :

The Conspirators draw, and kill Martius, who falls ; Aufidius stands on him

[...] Third Lord : Tread not upon him

(V, vi, 132).

Ailleurs ${ }^{15} \mathrm{j}^{\prime}$ 'ai montré, je l'espère, qu'à travers la folie de Lear, on se trouve confronté à un croisement entre les deux catégories du cannibalisme distinguées par les contemporains de Shakespeare - la rage canine et la fureur cannibale ; dans la tyrannie de Lear se profile un retour aux sources politiques de la tyrannie, au paradigme platonicien du concept, c'est-à-dire au spectacle sanguinaire du Temple de Zeus Lyceos dans le huitième livre de la Republique, où le gouverneur qui, ayant "gouté d" une langue, $d$ " une bouche impie "au sang familial" [...] bannit ou met $d$ mort [...et finit par] se muer en loup» (lyceos) atteint en d'autres termes d'une forme sacrilège de ce que les Elisabéthains auraient senti comme une espèce de «rage canine», incarné par le personnage du Scythe. J'ai dit déjà que la folie de Lear constitue une représentation alimentaire de l'inceste d'Edipe. Or, comme je l'ai 
rappelé, le nom d'Edipe a une face humaine, manifeste, exprimant l'omniscience de celui qui seul a su répondre à l'énigme du Sphinx, oida, je sais. L'autre face est la face voilée, divine, celle du savoir essentiel - de l'identité même de celui qui cherche à savoir et qui est l'objet recherché : oidos pous, pied enflé.

Qu'en est-il de Lear? De ce nom on peut dire deux choses : la première a trait au sens, la seconde à la matière phonique qui le compose ${ }^{16}$. En effet, de l'anglosaxon laernes au leery du dix-neuvième siècle (et même, dans le sud-ouest du vingtième) en passant par le leir élisabéthain (sans parler del'allemandleer) la même racine indo-européenne a fourni non seulement le nom du roi de la légende (dans la source, il s'écrit Leir, et il en existe des versions dans toute l'aire indo-européenne) mais aussi, dans toutes ces langues, avec des décalages diachroniques inévitables, le signifiant par excellence du manque. Or, ce nœud sémantique est important non seulement dans l'articulation obscure du désir mais déjà dans une lecture de surface de la pièce. En effet, le souhait aberrant de Lear «unburthen'd [to] crawl toward death» $(\mathrm{I}, \mathrm{i}, 41)$ réalise le sens propre du nom propre : à l'époque leir signifie «sans charge», et se dit notamment d'une bête de somme. Ainsi, étant sans charge, Lear entend être à charge. A l'époque élisabéthaine, l'antonyme de leir, c'était sumpter («portant fardeau»). Ainsi sert-il à Lear à définir l'image la plụs insoutenable qu'il puisse se faire de son sort : «Return with her !/Persuade me rather to be a slave and sumpter/To this detested groom !» (II, iv, 217).

Le seul sens de lear qui existe jusqu'à nos jours est celui qui se rencontre dans le sud-ouest et a été immortalisé par l'héroïne de The Mayor of Casterbridge de Hardy. Ce mot marque la séparation d'avec son père et précipite le drame :

Well, where have you been? he said to her with off-hand laconism.

"I've been strolling in the Walks and churchyard, father, till I feel quite leery". She clapped her hand to her mouth, but too late.

This was just enough to incense Henchard after the other crosses of the day. "I won't have you talk like that!" he thundered. "Leery», indeed. [...] if it goes on, this house can't hold us two.

«I feel leary», je meurs de faim. Bref, lear est le signifiant non seulement du manque mais du manque alimentaire poussé a la limite de l' inanition.

Le mot apparaîtau moins trois fois chez Hardy, et ce n'est pas nous éloigner de Shakespeare que de nous pencher un instant sur lescontextes où il surgit. Ce terme est doué d'une puissance d'évocation actuellement à la limite de l'oubli. A commencer par la glose du narrateur du Mayor of Casterbridge: «One failing of Elizabeth's was her occasional [...] use of dialect words - those terrible marks of the beast to the truly genteel» (ch. 20, our italics). Dans le poème «The BrideNight Fire», le mot est appelé par le désarroi et la solitude du héros qui, comme Lear, erre sur la lande à la limite de l'inconscience, puisque sa bien-aimée l'a rejeté 
The Lover Tim Tankens mourned heart-sick and leer

To be thus of his darling deprived.

He roamed in the dark ath' art field, mound and mere, [...].

Dans Tess of the d'Urbervilles, enfin, la scène où s'emploie ce terme se passe au milieu de la nuit, en rase campagne, hors des chemins battus, où, un peu comme dans The Faery Queene, l'homme rencontre la bête - il s'agit d'un taureau - et les deux se livrent jusqu'à l'aube à une lutte sans merci : «and 'twas only three o' clock in the world, and 'a knowed that nobody would come that way for hours, and he so leery and tired that " $a$ didn't know what to do [...] about four o' clock he felt that he verily would have to give over soon, and he said to himself, '[...] Heaven save me, or I'm a done man'». Ces trois occurrences sont éclairantes en ce qu'elles gardent toutes les trois les vestiges de cette situation-limite sous le signe du manque, où la faim verse dans l'inanition, où la privation bascule dans l'errance de l'exclusion voire, où l'être humain confine à la bête.

Aussi ce signifiant par excellence du manque joue-t-il à des niveaux différents pour créer un réseau qui sous-tend toute l'œuvre :

1. Comme LEIR, signe de la méconnaissance : c'est l'être «sans charge» à charge, qui, ayant démembré le corps de la terre mère nourricière voit son propre corps prêtà être englouti par le «sulphurus pit» du sexe de la femme, transformé en bouche non de l'apaisement ( I thought to set my rest on her kind nursery»), mais en celle de l'enfer et du tourment éternel, («there's hell, there's darkness»).

2. comme LEE/AR(Y), signe du manque alimentaire d'autant pius forclos que fondamental. Dans «The Bride-Night Fire», Hardy définit le terme dialectal leer en note comme «empty-stomached»; au début de l'acte III de Lear le gentilhomme évoque, comme doubles proléptiques de Lear lui-même, deux bêtes, l'une mâle, l'autre femelle, dont on pourrait dire qu'elles sont l'incarnation même de ce que c'est pour Hardy et Shakespeare d'être leer ou leery : «the belly-pinched wolf», le loup, emblématique comme on l'a déjà vu, et «the cub-drawn bear» : «this night, wherein the cub-drawn bear would couch, / The lion and the belly-pinched wolf / Keep their fur dry, unbonneted he runs» ; ce qui permet de faire le parallèle suivant, qui est loin de représenter simplement un jeu paronomastique :

Oidos pous, le roi pied crevé qui enfle

King Lear, le roi ventre creux qui gonfle.

En effet, la cohérence de la logique fantasmatique est telle que l'on peut dire que l'hysterica passio du vieux roi rejoint le plus ancien de tous les traités médicaux qui existent sur l'hystérie, le Papyrus Kahun, qui date d'environ 1900 av. J.C., et qui fut repris ensuite par les Grecs, Hippocrate notamment dans De morbis mulierum, où le mal est conçu en termes spécifiquement oraux-génitaux de «starvation of the womb» ${ }^{17}$. 
J'ai commencé cette étude en évoquant le premier des deux longs récits que contient le premier acte d'Hamlet, et les rapports créésà cette occasion entre nomos, ius gentium d'une part, remembrement des terres de l'autre, opposé à une imago maternelle effrayante. Cest par le second long récit, celui du fantôme que nous retrouverons le nom.

Le meurtre du roi gisant à même le sol dans son verger prend la forme d'un viol auriculaire. Ce viol auriculaire est, me semble-t-il, une triple parodie. La première se joue sur fond d'une topologie théologico-politique qui s'articule en termes d'axes, vertical et horizontal. Le liquide mortifere versé d'une fiole sur la tête horizontale du roi est une parodie du rituel de l'onction du roi (décrite par $\mathbf{M}$. Bloch ${ }^{18}$ ), cérémonie où le chrême miraculeux versé de la Sainte Ampoule sur la tête royale instaure la médiation verticale entre divinité et humanité.

Dans cette perspective, jusqu'à l'habit noir corbeau de Luciano est une inversion symétrique de la blanche colombe du Saint Esprit, censée transporter la fiole pour les besoins de la cause ( $«$ Come, the croaking raven doth bellow for revenge...»). Au lieu d'instaurer la médiation verticale, doublant le king body natural d'un king body politic, l'inversion horizontale du rituel au contraire sépare les deux corps du roi.

Par ailleurs, en tant qu' il affecte le king body natural, le viol auriculaire est une parodie à la fois de la Chute d'Eve dans le verger d'Eden et en même temps de 1'Annonciation faite à Marie, cette seconde Eve, selon les Pères de l'Eglise'19, destinée précisément à annuler la Chute. Ainsi, dans cette scène de viol auriculaire, scène qui est une véritable apothéose de l'imaginaire, le double corps de la femme ancêtre (bonne et mauvaise) surgit des décombres du symbolique (la parodie de la Sainte Onction) tel un phénix triompharit des flammes :

'Tis given out, that sleeping in my orchard, A serpent stung me - so the whole ear of Denmark Is by a forged process of my death Rankly abus'd

$$
(\mathrm{I}, \mathrm{v}, 35-8)^{30} \text {. }
$$

Or, quand cette représentation du viol auriculaire est revécue «en direct», au beau milieu de la pièce, c'est la souricière, mousetrap, autant que Le Meurtre de Gonzague, qui occupe le devant de la scène. Car l'abîme qui structure le texe en son centre est double, l'abîme du sexe se profilant derrière l'abyme du texte - the mousetrap avait un sens obscène au 16ème siècle. D'ailleurs, il convient de donner toute son importance à cette clé fournie par Hamlet le fils. En effet, au fond de l'abyme, Shakespeare nous donne à voir non pas une mais en réalité deux pièces : l'une jouée par les acteurs (Le Meurtre), l'autre jouée dans les intervalles de la première par Hamlet et Ophélie, et qui a pour titre «La Souriciere». Celle-ci est une mise à nu du fantasme originaire dans toute sa splendeur où le voyeurisme («I could 
interpret between you and your love if I could see the puppets dallying») se renverse aussitôt en exhibitionnisme ( $₫$ Be not you ashamed to show, he'll not shame to tell you what it means»). Shakespeare nous fait passer ainsi de la scène thêâtrale à l'autre scène, de la fiction au fantasme, de la fin aux origines, d'un excès d'hiératisme à un excès d'obscénité, du sublime au grotesque : du Meurtre de Gonzague à la Souriciere.

Où est le nom dans tout cela ? Or, qu'est-ce en effet que ce nom d'Hamlet, placé en titre au-dessus de ce spectacle d'un triple viol, d'un double abyme?

Dans le kenning, cette figure périphrastique, énigmatique, qui émaille la poésie nordique de l'Edda (Skaldsparmál, 23, 94) ${ }^{20}$, Amloai, c'est le trou dans la mer qui produit le blé nourricier: le sable en effet est évoqué, comme «la farine d'Amloai». Car Amloai est un moulin marin, un tourbillon énorme - un maëlstrom. Dans Hamlet, c'est autour de ce trou polysémique que gravitent les personnages, guetteurs transformés aussitôt en guettés, espions devenus histrions, etainsi de suite, selon la chorégraphie classique du fantasme. L'inquiétante étrangeté qui marque la pièce dès le lever du rideau trouve sa résolution et prend sa source dans l'énoncé énigmatique du kenning islandais dont le signifiant onomastique se révèle être une représentation grandiose - peut-être la plus grandiose - du paradigme freudien de l'Unheimlich: le trou dans la mer(e) nourricière qui vous engloutit ou vous recrache ${ }^{21}$.

Dans Othello, la tragédie qui suit Hamlet, 1604, les données se conforment à la même logique du signifiant onomastique même si, à la surface du texte, tout paraît très éloigné. Comme je l'ai suggéré ailleurs ${ }^{22}$, tout tourne, en dernière analyse, autour de l'effet de l'envie que Shakespeare désigne sous l'expression hautement évocatrice de «délacement du nom» (unlacing). Il s'agiten un premier temps du nom au sens figuré, c'est-à-dire du re-nom cher à Volumnia :

Othello : $[\ldots]$ What's the matter,

That you unlace your reputation thus,

And spend your rich opinion for the name

Of a night brawler?

(II, iii, 184-7) $)^{23}$.

Mais dans un deuxième temps ce «délacement» prend un sens littéral, car c'est le produit usuel d'un ultime délacement $d u$ corps - le sexe de la femme, la bocca vulvae, les «levres roses» - qui est dévoilé à mesure que le délire prend la place du désir dans l'esprit du héros : «Patience, thy young and rose-lipp'd cherubin, /I here 
look grim as hell» (IV, ii, 64-5). A ce moment-là, le nom dont il est question est le nom premier, le nom propre. En effet, la stratégie d'Iago face à l'héroïne revient en fait à faire surgir derrière le nom de la virginale, voire mariale Desdémone un autre nom, masqué, mauvais, maudit, qui fait retour des lointaines origines italiennes. Ce dernier est produit par «délacement» non des voiles du corps mais du signifiant onomastique lui-même, en enlevant la suture des deux faces qui le composent, donc par clivage et renversement des deux parties constituantes. Ainsi, en passant par Cinthio, chez qui Shakespeare avait puisé les rudiments de l'intrigue, on revient à Dante et à la double représentation du démon de Dis (chez Cinthio, Desdemona est déjà Disdemona), qui efface définitivement la double divinité de l'héroïne : celle de Vénus et celle de Marie ${ }^{24}$. En d'autres termes, «the divine Desdemona» devient le Ver du Monde coincé jusqu'à la taille - comme Aaron supplicié - dans l'abîme étouffant du dernier cercle de l'Enfer, jadis si splendide, à présent si détestable : «s' $e i$ fu bel com' egli è ora brutto»/ $O$ thou black weed why art so lively faire? ».

Or dans L'Inferno de Dante il y a deux représentations inverses et complémentaires, l'une liminaire, l'autre terminale, de Desdemona revue et corrigée en Démon de Dis : la face orale est représentée par le monstre qui mange les pécheurs dans le cercle central, la face phallique par le monstrè qui monte la garde à l'entrée de Dis, ce qui est, dans la série des 34 chants, la seule vision que Virgile interdit à Dante - ce qui se comprend, car c'est de Méduse qu'il s'agit :

Volgiti indietro e tien lo viso chiuso; che se il Gorgon si mostra, et tu li vedessi, nulla sarebbe del tornal mai suso

C'est cette représentation enfouie de Desdémone comme Disdemonio - comme Gorgone - qui explique pourquoi Othello ne suit pas les recommandations d'Iago, et, au lieu de l'étrangler, l'étouffe à l'aide d'un oreiller : pour se protéger du visage fatal, dont la beauté a été changée en horreur. Car c'est d'avoir été trop belle que Gorgô s'est retrouvée d'une laideur mortelle, et Persée lui-même, dans certaines versions du mythe, l'avait épousée avant de la tuer. Enfin, ce renversement du signifiant - cette antiphrase - se reflète en miroir dans le personnage qui sert de repoussoir à Desdémone : elle s'appelle Bianca, et elle est courtisane.

Dans $C$ dipe Roi, le nom d'CEdipe «délacé» livre l'inscription sur le corps $d u$ héros innocent d'un autre hiéroglyphe : oidos pous, le pied enflé dont l'autre face se révèle être le pied percé, troué, de l'innocent livré à la mort ; de même, chez Shakespeare, le délacement opéré par la logique de la représentation tragique sur le nom nous livre les deux faces du Démon de Dis : respectivement, la Gorgone phallique devant le premier cercle de Dis, et le monstre omophage du gouffre qui est au centre de la topologie dantesque du dernier cercle. Seulement chez Shakespeare, l'innocent n'est pas autre, il est un autre : un être dont le nom, à la fois icône et signe, fournit dans ses lettres une image de l'Enfer circonscrit par le premier et le 
dernier cercle : $O-\left(t h^{\prime}\right)$ hell-o.

Or, ce nom corporel n'est pas un simple hapax imaginaire livré par le travail interne au texte shakespearien. Un jour que je cherchais tout à fait autre chose, je suis tombée sur ce qui me paraît être l'acte équivalent figural de cette représentation onomastique, qui serait ainsi attesté dans le vocabulaire iconographique du Moyen Age anglais, ses racines remontant au moins jusqu'au quatrième siècle avant Jésus Christ. Il s'agit d'une image établie en Angleterre dans une série de manuscrits qui s'attachent à St. Albans, dont le scriptorium a été administré par Mathieu Paris, auteur d'un inventaire des pierres antiques si prisées au Moyen Age et à la Renaissance. Dans les premières apocalypses anglo-normandes en effet, le gouffre de l'enfer est représenté sous les espèces d'un gorgoneïon doublé, analogue à celui répertorié sur un scarabée de Tharros ${ }^{25}$, où les deux gueules sontétirées pour dévoiler dans l'abîme ainsi découvert le spectacle des tourments éternels. Ce n'est pas un hasard, me semble-t-il, si le travail de sape du verbe satanique d'Iago, de cette parole empoisonnée de la calumnia tant redoutée au Moyen Age et à la Renaissance (qui la concevaient comme une réalité physiologique de subversion du corps politique et naturel), en abolissant le nom du Père, fait surgir du tréfonds de l'âme européenne ce superbe exemple de sycrétisme eschatologique où l'épouvante chrétienne et l'épouvante antique s'enchâssent l'une dans l'autre, et l'enfer s'encadre de la gueule de Gorgô.

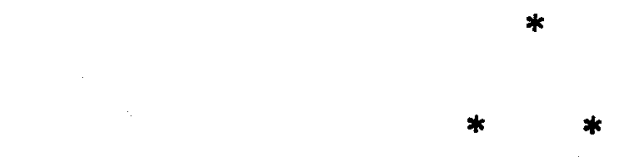

L'effacement du Nom-du-Père est ainsi la clé-de-voûte linguistique de ces textes, et on comprend pourquoi Saint Augustin insistait sur l'efficacité du baptême (l'un des deux seuls sacrements retenus par l'Eglise anglicane) comme traitement préventif contre l'envie, dont il a fait une analyse, reprise par Lacan, en termes de frustration du sein maternel. Le rapport entre le Nom-du-Père et le nom monstrueux de la Mère, ou si vous voulez, du nom diabolique, un nom en tout cas qui est l'image de l'objet du désir et non l'expression de son inaccessibilité en fonction du partage coutumier des mères autant que des terres, un tel rapport est fondamental tant dans la théologie chrétienne que dans la tragédie shakespearienne. 


\section{NOTES}

1 Paris, Fata Morgana, 1976.

2 Op.cit. p. 8.

3 Oxford, O.U.P. 1984, p. 24.

4 Cf. notamment l'essai de J.-P. Vernant «Ambiguïté et renversement. Sur la structure énigmatique d'Edipe Roi» dans Mythe et Tragédie en Grèce Ancienne», Paris, Maspéro, 1972.

5 Comme l'anamorphose de Holbein abolit les Ambassadeurs.

6 Toutes références à l'édition Arden, ed. P. Brockbank, Londres, Methuen, 1976.

7 J.-A. Miller, Ornicar? Janvier 1975, p. 26.

8 Toutes références à l'édition Arden, ed. Stanley Wells, Londres, Methuen, 1982.

9 Toutes références à l'édition Arden, ed. Kenneth Muir, Londres, Methuen, 1982.

10 Toutes références à l'édition d'I. Gollancz, Londres, Dent, s.d. Je souligne.

11 Comme le précise D. Jones dans le English Pronouncing Dictionary, Londres, Dent, (1917), 1964, Aaron se prononce comme airlearan/ en anglais ; la prononciation contemporaine des Américains /æeran/n'est pas pertinente ici.

12 Op. cit. p. 41.

13 Cf. L'article de D. J. Gordon, «Name and fame» in Coriolanus, dans Papers Mainly Shakespearian, ed. G.I. Duthie, Londres, 1964.

14 Il semble y avoir un rapport entre le nom et le sang qui coule, l'oubli du nom se produisant alors que le sang ne coule plus sur son corps et que, faute de ce breuvage enivrant, force lui est de demander ce qui en est le substitut consacré - le vin, emblème du Nom sacré de Dieu lors du mystère de la transubstantiation :

Lartius : Martius, his name?
Cor. :
By Jupiter, I forgot!
I am weary, yea, my memory is tired; Have we no wine here?
Cominius :
Go we to our tent.
The blood upon your visage dries.

(I, ii, 87-90).

15 Dans une communication faite au colloque «Raison et folie» de la Société Shakespeare, nov. 1985. Voir aussi «Le Roi Lear ou la Tyrannie entre Platon et Strindberg» à paraître dans Théâtre public.

16 Je n'ai pas la place pour traiter de cet aspect ici.

17 Cf. Veith, Iza, Hysteria : the History of a Disease, Chicago, 1965.

18 DansLes Rois Thaumaturges de MarcBloch, (1924)Paris, Gallimard, 1983 : avant tout peut-être, l'image du roi ennemi de la maladie des écrouelles transformé lui-même en malade atteint d'une corruption de la peau apparemment identique à celle qu'habituellement - en signe de légitimité royale - il guérit.

19 Cf. St. Ephrem : «Au commencement le serpent prit possession des oreilles d'Eve, et de là infusa son venin dans tout son corps; aujourd' hui Marie a reçu par ses oreilles le 
Champion de la béatitude universelle».

20 Cf. The Commentary to Saxo Grammaticus History of the Danes, de Peter Fisher, Cambridge, Brewer, 1980, vol. II, p. 59.

Autre exemple de la même figure, qui utilise la même image du moulin, l'or est évoqué dans d'autres Kenningar comme «la farinelle blé de Frodi», ce demier, l'un des premiers rois légendaires du Danemark, possédait un moulin nommé Grotti qui produisait tout ce qu'on lui demandait, et notamment de l'or, première substance à en sortir.

Les kenningar du Viel Nordique étaient extrêmement complexes, bien plus que ceux de la poésie en ancien anglais (swanard, route des cygnes, donc «la mer»; helmberend, celui qui porte le heaume, donc «guerrier»).

21 J.-M. Maguin rappelle pertinemment que quand Hamlet voit le fantôme, il n'est pas du tout sûr qu'il s'agisse de son père, bien qu'il lui en donne le titre. La réponse est l̄a suivante : mon propos dans cette étude concerne le signifiant en tant que tel, non le référent - ou plus exactement le signifiant pour autant qu'il est instable, pris précisément dans un glissement sur le signifié ; que Hamlet dise au fantôme l «Qui êtes-vous ? Je veux bien vous nommer Hamlet», indique on ne peut plus clairement que le rapport (saussurien) S/s, qui constitue le signe linguistique, ici ne fonctionne plus. Jean Paris dans Hamlet et Panurge parle du dépassement du système (médiéval) des «signatures» dans la pièce : au-delà des signatures, il s'agit du signè lui-même pour autant qu'il se compose d'un signifiant et d'un signifié dont le rapport peut ne pas être biunivoque.

22 Dans «The Unlacing of the Name» dans Autour d'Othello», Amiens, 1987.

23 Toutes références à l'édition Arden, ed. M.R. Ridley, Londes, Methuen, (1958) 1982.

24 La fraise, la référence à la caraque, ainsi que divers discours, dont un qui est proche de l'Ave Maria, se conjuguent pour créer une aura sinon une auréole marialle. Le problème, c'est que la même fraise et le même discours peuvent être interprétés également comme emblème de Vénus, qui, dans la tradition du Moyen Age, était une incarnation de la Luxure.

25 Cf. Le Moyen Age fantastique de J. Baltrusaïtis, Paris, A. Colin 1955, p. 37. 